\title{
Isolation of Protein Tyrosine Phosphatase 1B Inhibitory Constituents from the Sclerotia of Polyporus umbellatus Fries
}

\author{
Hee Sang Lee, In Hyun Hwang, Jeong Ah Kim, Ji Young Choi, Tae-Su Jang, ${ }^{\dagger}$ Hiruyuki Osada, ${ }^{\dagger}$ Jong Seog Ahn, ${ }^{\S, *}$ \\ MinKyun Na, and Seung Ho Lee* \\ College of Pharmacy, Yeungnam University, Gyeongsan, Gyeongbuk 712-749, Korea \\ *E-mail: mkna@ynu.ac.kr (M. Na),seungho@ynu.ac.kr (S.H. Lee) \\ ${ }^{\dagger}$ College of Medicine, CHA University, Seoul 135-080, Korea \\ *The Institute of Physical and Chemical Research (RIKEN) Wako-shi, Saitama 351-0198, Japan \\ ${ }^{\S}$ Korea Research Institute of Bioscience and Biotechnology (KRIBB), Daejeon 305-333, Korea. *E-mail: jsahn@kribb.re.kr \\ Received August 9, 2010, Accepted November 24, 2010
}

Key Words: Protein tyrosine phosphatase 1B, Polyporus umbellatus, Ceramide, Steroid

Recently, interest in specific and potent protein tyrosine phosphatase $1 \mathrm{~B}$ (PTP1B) inhibitor that key regulator both in insulin and leptin signaling pathways is rising up. ${ }^{1-3} \mathrm{PTP} 1 \mathrm{~B}$ is an important negative modulator in insulin signaling pathway. ${ }^{4-6}$ So it is considered as a good target for the treatment of type 2 diabetes and obesity. ${ }^{7}$ To date, several types of PTP1B inhibitors have been developed. However, most of them have a problem on the side effects and low bioavailability, which facilitate investigation on natural products to find out safe and drug-like PTP1B inhibitors. $^{8,9}$

In the course of our screening program for medicinal plants, using in vitro PTP1B enzyme assay, a $\mathrm{MeOH}$ extract of the sclerotia of Polyporus umbellatus Fries (Polyporaceae) showed PTP1B inhibitory activity at a level of $30 \mu \mathrm{g} / \mathrm{mL}$. The sclerotia of $P$. umbellatus is a saprophytic mushroom that grows on the roots of Alnus sp., Quercus sp., and Betula sp. Traditionally, this crude drug has been used as a diuretics, ${ }^{10}$ antipyretics, ${ }^{11}$ and anti-inflammatory ${ }^{12}$ agent. There have been many reports on the constituents isolated from the sclerotia of $P$. umbellatus. Among them, steroids such as ergosterol, ${ }^{13}$ ergosta-4,6,8(14), 22-tetraen-3-one, ${ }^{14}$ and polyporusterones $\mathrm{A} \sim \mathrm{G}^{15}$ are representative constituents of this species. Glucan ${ }^{16}$ and fatty acids were also isolated from the species. Although a number of studies on the sclerotia of $P$. umbellatus have been conducted, the PTP1B inhibitory activity and its active constituents have not demonstrated yet. In our current study, we found out that some steroids and a new ceramide isolated from the sclerotia of $P$. umbellatus had the PTP1B inhibitory activity. In this paper, we describe the isolation, and structure determination of a new ceramide $(2 S$, $\left.3 S, 4 R, 2^{\prime} R\right)-2-\left(2^{\prime}\right.$-hydroxytricosanoylamino) nonadecane-1,3,4triol (1) (Fig. 1) as well as the inhibitory activity of isolates against PTP1B. In addition, we report on the isolation of steroids, 19-norergosta-5,7,9,22-tetraene-3 $\beta$-ol (2), ${ }^{17} 24$-ethylcholesta-7,22-diene-3 $\beta, 5 \alpha, 6 \beta$-triol (3), ${ }^{18,19} 24$-methylcholesta7,22-diene-3 $\beta, 5 \alpha, 6 \beta$-triol (4), ${ }^{20,21}$ ergosterol peroxide (5), ${ }^{22}$ ergosta-7,22-diene-3 $\beta$-ol (6), ${ }^{23}$ and ergosta-7,22-diene-3-one $(7)^{24}$ (Fig. 1), and their PTP1B inhibitory activity.

Compound 1 was obtained as a white powder with an optical rotation value, $[\alpha]_{\mathrm{D}}^{21}+47.7$ ( $c 0.05$, pyridine). The HRFABMS spectrum of 1 indicated the molecular formula $\mathrm{C}_{42} \mathrm{H}_{85} \mathrm{NO}_{5}$
$\left([\mathrm{M}+\mathrm{H}]^{+}\right.$684.6508, calcd. 684.6506). The ${ }^{1} \mathrm{H}-\mathrm{NMR}$ spectrum of 1 showed the presence of two terminal methyl groups at $\delta_{\mathrm{H}}$ $0.88(3 \mathrm{H}, \mathrm{t}, J=4.8 \mathrm{~Hz}), 0.87(3 \mathrm{H}, \mathrm{t}, J=4.8 \mathrm{~Hz})$, methylene protons at $\delta_{\mathrm{H}} 1.27 \sim 1.40(60 \mathrm{H}$, br s) indicating a long alkyl chain. A pair of oxymethylene proton (H-1) peaks were assigned at $4.52(1 \mathrm{H}, \mathrm{dd}, J=4.6,10.8 \mathrm{~Hz}, \mathrm{H}-1 \beta), 4.43(1 \mathrm{H}, \mathrm{dd}, J=4.9$, $10.8 \mathrm{~Hz}, \mathrm{H}-1 \alpha)$, three oxygenated methine protons (H-2', 3, 4) were appeared at $\delta_{\mathrm{H}} 4.63\left(1 \mathrm{H}, \mathrm{dd}, J=3.5,7.5 \mathrm{~Hz}, \mathrm{H}-2^{\prime}\right), 4.36$ $(1 \mathrm{H}, \mathrm{t}, J=5.6 \mathrm{~Hz}, \mathrm{H}-3), 4.30(1 \mathrm{H}, \mathrm{m}, \mathrm{H}-4)$, and an amide proton signal at $8.58(1 \mathrm{H}, \mathrm{d}, J=9.0 \mathrm{~Hz})$. A multiplet signal at $\delta_{\mathrm{H}}$ $5.11(1 \mathrm{H}, \mathrm{m})$ was assigned as a methine proton next to the nitrogen atom. These ${ }^{1} \mathrm{H}-\mathrm{NMR}$ data suggested that 1 should be a phytosphingosine-type ceramide containing a 2-hydroxy fatty

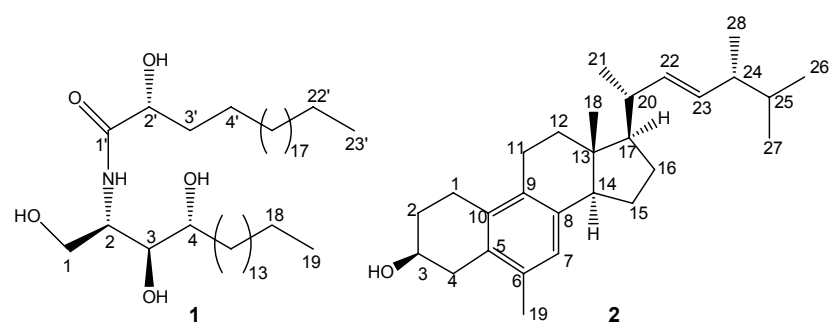

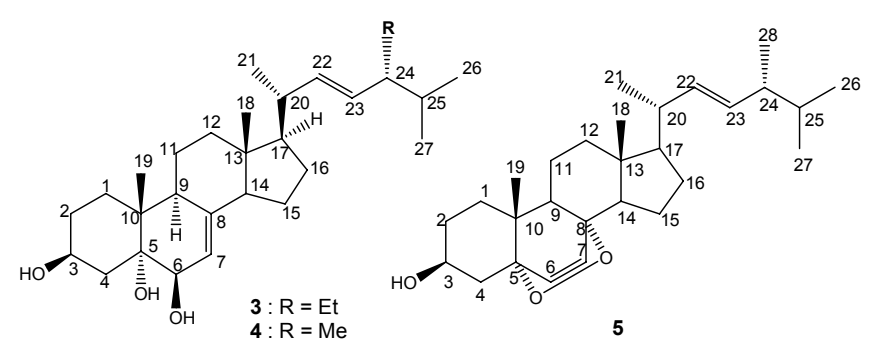

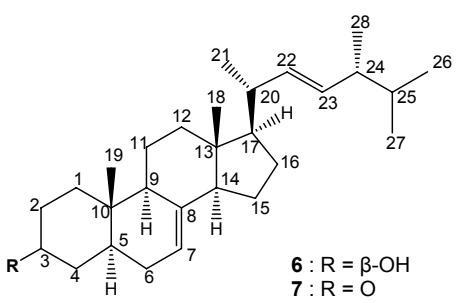

Figure 1. Compounds 1-7 isolated from the Sclerotia of $P$. umbellatus 
acid. ${ }^{25,26}$ The ${ }^{13} \mathrm{C}-\mathrm{NMR}$ spectrum of $\mathbf{1}$ showed the signal for carbonyl group at $\delta_{\mathrm{C}} 175.3$, three oxymethines at $\delta_{\mathrm{C}} 76.8(\mathrm{C}-3)$, 73.1 (C-4), and 72.4 (C-2'). In the HMBC spectrum, H-1 [ $\delta_{\mathrm{H}} 4.52$ $(1 \mathrm{H}, \mathrm{dd}, J=4.6,10.8 \mathrm{~Hz}, \mathrm{H}-1 \beta), 4.43(1 \mathrm{H}, \mathrm{dd}, J=4.9,10.8$ $\mathrm{Hz}, \mathrm{H}-1 \alpha)]$ correlated with $\delta_{\mathrm{C}} 52.9(\mathrm{C}-2), 76.8(\mathrm{C}-3)$, and $\delta_{\mathrm{H}} 4.36$ $(1 \mathrm{H}, \mathrm{t}, J=5.6 \mathrm{~Hz}, \mathrm{H}-3)$ also correlated with C-2. Furthermore, the amide proton at $\delta_{\mathrm{H}} 8.58(1 \mathrm{H}, \mathrm{d}, J=9.0 \mathrm{~Hz})$ correlated with $\mathrm{C}-2$, which indicated the imine carbon is $\mathrm{C}-2$. Concomitant analysis of the COSY, HMQC, and HMBC data led to the determination of partial structure as a ceramide. The relative configuration of 1 , in particular, the position of C-2, C-3, C-4 and $\mathrm{C}-2^{\prime}$ was identified to be the same as those of natural and synthetic known ceramide, $\left(2 S, 3 S, 4 R, 2^{\prime} R\right)-2-\left(2^{\prime}\right.$-hydroxytetracosanoylamino)octadecane-1,3,4-triol, on the basis of both the chemical shifts and coupling constants for $\mathrm{H}-2, \mathrm{H}-3, \mathrm{H}-4$, and $\mathrm{H}-2^{\prime}$. The optical rotation value of $\mathbf{1}$ was similar to those of known compound from natural sources $\left([\alpha]_{D}=+11.5^{\circ}\right)$ and synthetic one $\left([\alpha]_{D}=+9.1^{\circ}\right)$, which suggested that 1 has the same absolute configuration as known compound. ${ }^{27,28}$ The aspect of biogenetic considerations, which this type of ceramides has been reported to be a unique class of secondary metabolites and isolated from a number of marine organisms, ${ }^{25,27}$ fungi ${ }^{26}$ and mushrooms, ${ }^{28}$ promises strong conclusion to have $\left(2 S, 3 S, 4 R, 2^{\prime} R\right)$ sphingosine moiety in $\mathbf{1}$, despite of the slight difference with their structures. Although most of the NMR data for 1 were quite similar to those of known ceramide, some of the MS data indicating a fragmentation pattern were different from the known ceramide. In the FABMS spectrum of $\mathbf{1}$, some intensive ion signals were observed at $m / z 426,384,300$, and 282, which were in accordance with the fragmentation for $\left[\mathrm{M}+\mathrm{H}-\mathrm{CH}_{3}\left(\mathrm{CH}_{2}\right)_{13}\right.$ $\mathrm{CHOH}-\mathrm{OH}]^{+},\left[\mathrm{CH}_{3}\left(\mathrm{CH}_{2}\right)_{20} \mathrm{CHOHCO}-\mathrm{NHCH}_{3}\right]^{+},\left[\mathrm{CH}_{3}\left(\mathrm{CH}_{2}\right)_{14}\right.$ $\left.\mathrm{CHOHCH} \mathrm{OH}-\left(\mathrm{CH}_{2}\right)_{2} \mathrm{OH}\right]^{+}$, and $\left[\mathrm{CH}_{3}\left(\mathrm{CH}_{2}\right)_{13} \mathrm{CH}=\mathrm{CHCOH}=\right.$ $\left.\mathrm{CH}-\mathrm{NH}_{2}\right]^{+}$, respectively (Fig. 2). Methanolysis of 1 with $\mathrm{HCl}$ in $\mathrm{MeOH}$ afforded a fatty acid methyl ester. On the basis of a pseudo-molecular ion peaks at $m / z 383[\mathrm{M}-\mathrm{H}]^{-}$and $324[\mathrm{M}-$ $\left.\mathrm{H}-\mathrm{COOCH}_{3}\right]^{-}$in the ESIMS/MS as well as the characteristic ${ }^{1} \mathrm{H}$ NMR resonance at $\delta 0.86(\mathrm{t}, J=6.1 \mathrm{~Hz})$ and $3.77\left(\mathrm{~s}, \mathrm{OCH}_{3}\right)$, the hydrolyzed fatty acid methyl ester was confirmed as a methyl 2-hydroxytricosanoate. From the evidence, 1 was determined to be $\mathrm{C}_{23}$-phytosphingosine unit containing three hydroxyl

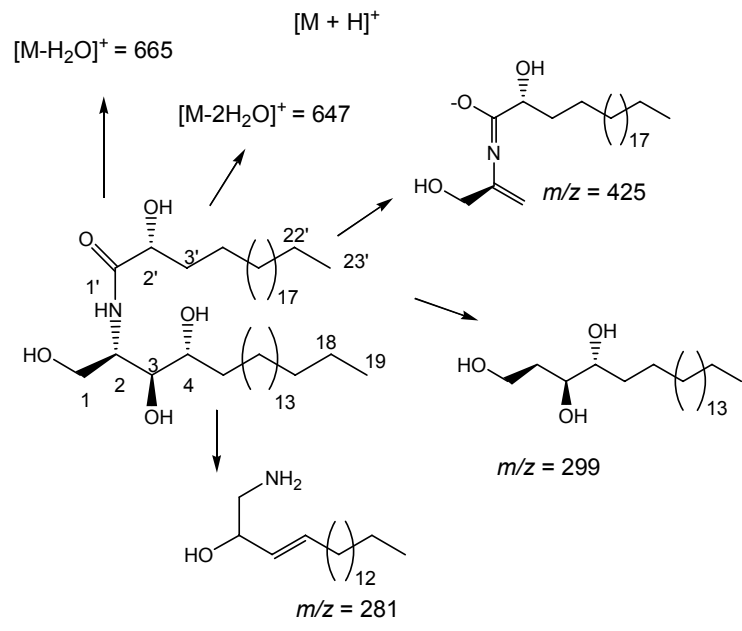

Figure 2. MS Fragmentation of $\mathbf{1}$ in FAB Mass Spectrum groups and $\mathrm{C}_{19}$-fatty acid. Consequently, 1 was characterized as $\left(2 S, 3 S, 4 R, 2^{\prime} R\right)-2-\left(2^{\prime}\right.$-hydroxytricosanoylamino) nonadecane1,3,4-triol, which was ascertained to be a new ceramide.

In the course of the phytochemical investigation, six steroids were isolated. The structures of compounds were identified as 19-norergosta-5,7,9,22-tetraene-3 $\beta$-ol (2), 24-ethylcholesta7,22-diene-3 $\beta, 5 \alpha, 6 \beta$-triol (3), 24-methylcholesta-7,22-diene$3 \beta, 5 \alpha, 6 \beta$-triol (4), ergosterol peroxide (5), ergosta-7,22-diene$3 \beta$-ol (6), and ergosta-7,22-diene-3-one (7) by physicochemical $\left([\alpha]_{\mathrm{D}}\right)$ and spectroscopic analysis (MS, NMR). Of the steroids isolated, compound $\mathbf{2}$ was isolated from the species for the first time.

All the compounds 1-8 were tested for their inhibitory activity against PTP1B. As shown in Table 2 , the new ceramide $(2 S$, $\left.3 S, 4 R, 2^{\prime} R\right)-2-\left(2^{\prime}\right.$-hydroxytricosanoyl-amino)nonadecane-1,3,4triol (1) showed marginal PTP1B inhibitory activity with $\mathrm{IC}_{50}$ value of $25.1 \pm 0.1 \mu \mathrm{g} / \mathrm{mL}$. Although, compounds 2-7 have a similar steroidal backbone, only 19-norergosta-5,7,9,22-tetraene-3 $\beta$-ol (2), 24-ethylcholesta-7,22-diene-3 $\beta, 5 \alpha, 6 \beta$-triol (3), and 24-methylcholesta-7,22-diene-3 $\beta, 5 \alpha, 6 \beta$-triol (4) showed high PTP1B inhibitory activity with $\mathrm{IC}_{50}$ values of $8.9 \pm 0.3$, $6.5 \pm 0.6$ and $7.5 \pm 0.2 \mu \mathrm{g} / \mathrm{mL}$, respectively, whereas compounds 5-8 appeared to be inactive. The results indicate that active steroidal compounds (2-4) from the sclerotia of $P$. umbellatus can be regarded as sources for the PTP1B inhibitors, which are prompted by previous reports on steroids of their pharmacological actions. Steroids isolated from plants have been reported to inhibit the formation of nitric oxide (NO) in the lipopolysaccharide (LPS)-activated murine macrophage. ${ }^{29}$ Steroids have anti-microbial activity and can be applied as adjuvant in anti-

Table 1. ${ }^{1} \mathrm{H}$ and ${ }^{13} \mathrm{C}$ NMR data of compound $\mathbf{1}$ (pyridine- $d_{5}$ )

\begin{tabular}{lll}
\hline position & \multicolumn{1}{c}{$\delta_{\mathrm{C}}{ }^{a}$} & $\delta_{\mathrm{H}}$, mult. $(J \text { in Hz})^{b}$ \\
\hline $\mathrm{NH}$ & & $8.58, \mathrm{~d}(J=9.0 \mathrm{~Hz})$ \\
$1 \alpha$ & 62.0 & $4.43, \mathrm{dd}(J=4.9,10.8 \mathrm{~Hz})$ \\
$1 \beta$ & & $4.52, \mathrm{dd}(J=4.6,10.8 \mathrm{~Hz})$ \\
2 & 52.9 & $5.11, \mathrm{~m}$ \\
3 & 76.8 & $4.36, \mathrm{t}(J=5.6 \mathrm{~Hz})$ \\
4 & 73.1 & $4.30, \mathrm{~m}$ \\
$5 \alpha$ & 34.2 & $1.94, \mathrm{~m}$ \\
$5 \beta$ & & $2.01, \mathrm{~m}$ \\
6 & 26.7 & $1.27-1.40, \mathrm{br} \mathrm{s}$ \\
$7-16$ & $30.0-30.4$ & $1.27-1.40, \mathrm{br} \mathrm{s}$ \\
17 & $32.1^{c}$ & $1.27-1.40, \mathrm{br} \mathrm{s}$ \\
18 & $22.9^{c}$ & $1.27-1.40, \mathrm{br} \mathrm{s}$ \\
19 & 14.3 & $0.88, \mathrm{t}(J=4.8 \mathrm{~Hz})$ \\
$1^{\prime}$ & 175.3 & \\
$2^{\prime}$ & 72.4 & $4.63, \mathrm{dd}(J=3.5,7.5 \mathrm{~Hz})$ \\
$3^{\prime} \alpha$ & 35.7 & $2.13, \mathrm{~m}$ \\
$3^{\prime} \beta$ & & $2.26, \mathrm{~m}$ \\
$4^{\prime}$ & 25.8 & $1.79, \mathrm{~m}$ \\
$5^{\prime}-20^{\prime}$ & $30.0-30.4$ & $1.27-1.40, \mathrm{br} \mathrm{s}$ \\
$21^{\prime}$ & $32.1^{c}$ & $1.27-1.40, \mathrm{br} \mathrm{s}$ \\
$22^{\prime}$ & $22.9^{c}$ & $1.27-1.40, \mathrm{br} \mathrm{s}$ \\
23 & 14.2 & $0.87, \mathrm{t}(J=4.8 \mathrm{~Hz})$ \\
\hline
\end{tabular}

${ }^{a}$ Assignments based on HMQC and HMBC data $(150 \mathrm{MHz}) .{ }^{b}$ Assignments based on COSY and HMBC data $(600 \mathrm{MHz}) .{ }^{c}$ Assignments may be interchanged. 
Table 2. $\mathrm{IC}_{50}$ Values of Isolated Compounds from the Sclerotia of $P$. umbellatus in PTP1B Inhibitory Activity Assay

\begin{tabular}{|c|c|}
\hline Compound & $\begin{array}{l}\text { PTP1B inhibition } \\
\quad\left(\mathrm{IC}_{50} \mu \mathrm{g} / \mathrm{mL}\right)^{a}\end{array}$ \\
\hline $\begin{array}{l}\left(2 S, 3 S, 4 R, 2^{\prime} R\right)-2-\left(2^{\prime} \text {-hydroxytricosanoylamino)- }\right. \\
\quad \text { nonadecane-1,3,4-triol (1) }\end{array}$ & $25.1 \pm 0.1$ \\
\hline 19-norergosta-5,7,9,22-tetraene-3 $\beta$-ol (2) & $8.9 \pm 0.3$ \\
\hline 24-ethylcholesta-7,22-diene-3 $\beta, 5 \alpha, 6 \beta$-triol (3) & $6.5 \pm 0.6$ \\
\hline 24-methylcholesta-7,22-diene- $3 \beta, 5 \alpha, 6 \beta$-triol (4) & $7.5 \pm 0.2$ \\
\hline ergosterol peroxide (5) & $>50$ \\
\hline ergosta-7,22-diene-3 $\beta$-ol (6) & $>50$ \\
\hline ergosta-7,22-diene-3-one (7) & $>50$ \\
\hline $\mathrm{RK}-682^{b}$ & $5.0 \pm 0.5$ \\
\hline
\end{tabular}

${ }^{a} \mathrm{IC}_{50}$ values of PTP1B inhibitory activity at $30 \mu \mathrm{g} / \mathrm{mL} .{ }^{b}$ Positive control.

parasitic chemotherapy as glutathione $S$-transferase inhibitor. ${ }^{30}$ They can reduce the LDL cholesterol level by controlling intestinal cholesterol absorption and reducing dietary and biliary cholesterol incorporation into micelles. ${ }^{31}$ Interestingly, steroids were demonstrated to have a blood glucose lowering effect, which might be associated with the PTP1B inhibitory activity. ${ }^{32,33}$ Although the PTP1B inhibition by steroidal compounds needs to be further investigated, this study suggests that the components from the sclerotia of $P$. umbellatus may be considerable for development as PTP1B inhibiting agents.

\section{Experimental}

General. Optical rotations were measured using a JASCO DIP-1000 (Tokyo, Japan) automatic digital polarimeter. The FT-IR spectra were recorded on a JASCO FT-IR 300E spectrophotometer, and UV spectra on a JASCO V-550 spectrophotometer. The NMR spectra were recorded on a Bruker $250 \mathrm{MHz}$ (DMX 250) spectrometer and a Varian 600 MHZ (VNS 600), respectively, using Bruker's and Varian's standard pulse program, with chemical shifts reported in ppm downfield from TMS. Column chromatography was carried out on Merck silica gel (70 - 230 mesh, Merck). TLC was performed on aluminum plates precoated with Kieselgel $60 \mathrm{~F}_{254}$ (Merck). All other chemicals and solvents were of analytical grade. The LCQ advantage trap mass spectrometer (Thermo Finnigan, San Jose, CA) was equipped with an electrospray ionization (ESI) source.

Plant Material. The dried sclerotia of $P$. umbellatus (Polyporaceae) was purchased from a folk medicine market "Yakryong-si" in Daegu, Korea, in June 2007, and identified by Prof. Seung Ho Lee (Yeungnam University, Korea). A voucher specimen (SH006-179) has been deposited at the College of Pharmacy, Yeungnam University, Korea.

PTP1B Assay. PTP1B (human, recombinant) was purchased from BIOMOL International LP (Plymouth Meeting, PA). The enzyme activity was measured $p$-nitrophenyl phosphate ( $p$ NPP). To each of 96 wells in a microtiter plate (final volume: $100 \mu \mathrm{L}$ ) was added $2 \mathrm{mM} p \mathrm{NPP}$ and PTP1B $(0.05-0.1 \mu \mathrm{g})$ in a buffer containing $50 \mathrm{mM}$ citrate ( $\mathrm{pH} 6.0$ ), $0.1 \mathrm{M} \mathrm{NaCl}, 1$ mM EDTA, and $1 \mathrm{mM}$ dithiothreitol (DTT), with or without test compounds. Following incubation at $37^{\circ} \mathrm{C}$ for $30 \mathrm{~min}$, the reaction was terminated with $10 \mathrm{M} \mathrm{NaOH}$. The amount of produced $p$-nitro- phenol was estimated by measuring the absorbance at $405 \mathrm{~nm}$. The nonenzymatic hydrolysis of $2 \mathrm{mM} p \mathrm{NPP}$ was corrected by measuring the increase in absorbance at $405 \mathrm{~nm}$ obtained in the absence of PTP1B enzyme.

Extraction and Isolation. The dried sclerotia of $P$. umbellatus Fries $(10 \mathrm{~kg})$ was extracted three times with $\mathrm{MeOH}$ at room temperature for three days. The $\mathrm{MeOH}$ solution was concentrated under reduced pressure to give a residue $(148 \mathrm{~g})$ and it was partitioned between $\mathrm{H}_{2} \mathrm{O}$ and ethyl acetate (EtOAc). A portion of the EtOAc fraction $(58 \mathrm{~g})$ was separated by silica gel column chromatography $(100 \times 9 \mathrm{~cm})$ eluting with a gradient of methylene chloride (MC) in EtOAc (99, 98, 95, 93, 90, 80, 50, and 0\%), to give 13 fractions (PE1 - PE13). The fraction PE13 (0.4980 g) was submitted to column chromatography over silica gel column $(50 \times 4 \mathrm{~cm}$ ) and eluted with MC-acetone (from 95:5 to 0:100) to give 8 fractions (PE13.1 - PE13.8). Compound 1 (23 mg), which was acquired from the subfraction PE13.6, was recrystallized with $\mathrm{MeOH}$. Seven fractions (PE12.1 - PE12.7) were isolated from fraction PE12 $(1.679 \mathrm{~g})$ through silica gel column $(50 \times$ $3 \mathrm{~cm}$ ) chromatography and eluted with $n$-hexane/acetone $(97: 3$, 95:5, 92:8, 85:15, 70:30, 50:50, 0:100). Subfraction PE12.5 $(0.823 \mathrm{~g})$ was isolated with silica gel column $(60 \times 3 \mathrm{~cm})$ chromatography (acetone $/ n$-hexane, $5-50 \%$ ) over again, resulting in 5 fractions (PE12.5.1 - PE12.5.5), from which subfraction PE12.5.3 $(0.6984 \mathrm{~g})$ was recrystallized in $\mathrm{MeOH}$ to afford compound $4(10 \mathrm{mg})$. Column chromatography of the $\mathrm{H}_{2} \mathrm{O}$ fraction (88 g) over silica gel, using $\mathrm{MeOH}(1-50 \%)$ in $\mathrm{MC}$ as solvent, yielded 13 fractions (PH1 - PH13), according to their TLC patterns. PH6 $(1.110 \mathrm{~g})$ after recrystallization was purified by silica gel column $(60 \times 5 \mathrm{~cm})$ chromatography, using acetone/ $\operatorname{MC}(0.2,0.4,0.6,1$, and $100 \%)$ as an eluting solution to ultimately produce 16 fractions (PH6.1 - PH6.16), subfraction PH6.4 was afforded to compound $2(50 \mathrm{mg})$. The fraction PH8 $(0.93 \mathrm{~g})$ was separated by column chromatography over silica gel column $(50 \times 4.5 \mathrm{~cm})$ and eluted with $\mathrm{MeOH}-\mathrm{MC}$ in a step gradient (2 - 50\%), yielding 6 fractions (PH8.1 - PH8.6). Compound 3 $(40 \mathrm{mg})$ was isolated from subfraction PH8.5. Four other compounds, ergosterol peroxide (5), ergosta-7,22-diene-3 3 -ol (6), ergosta-7,22-diene-3-one (7), and docosanoic acid (8), were also isolated from a $\mathrm{MeOH}$ extract of polyporus, using the silica gel column chromatography. The compounds structures (Fig. 1) are determined by ${ }^{1} \mathrm{H},{ }^{13} \mathrm{C}$, DEPT NMR and low, high FABMS analysis, and by comparison of their physical and spectroscopic data with those reported in the literature.

(2S,3S,4R,2'R)-2-(2'-Hydroxytricosanoylamino)nonadecane1,3,4-triol (1): White powder; $[\alpha]_{\mathrm{D}}^{21}+47.7^{\circ}(c 0.05$, pyridine); HR-FABMS $m / z[\mathrm{M}+\mathrm{H}]^{+}$calcd. $\mathrm{C}_{42} \mathrm{H}_{85} \mathrm{NO}_{5}: 684.6506$ Found 684.6508 FABMS $m / z 684[\mathrm{M}+\mathrm{H}]^{+}, 666\left[\mathrm{M}+\mathrm{H}-\mathrm{H}_{2} \mathrm{O}\right]^{+}, 648$ $\left[\mathrm{M}+\mathrm{H}-2 \mathrm{H}_{2} \mathrm{O}\right]^{+}, 572,558,526,426\left[\mathrm{M}+\mathrm{H}-\mathrm{CH}_{3}\left(\mathrm{CH}_{2}\right)_{13} \mathrm{CHOH}^{-}\right.$ $\mathrm{OH}]^{+}, 384\left[\mathrm{CH}_{3}\left(\mathrm{CH}_{2}\right)_{20} \mathrm{CHOHCO}-\mathrm{NHCH}_{3}\right]^{+}, 342,318,316$, $300\left[\mathrm{CH}_{3}\left(\mathrm{CH}_{2}\right)_{14} \mathrm{CHOHCHOH}-\left(\mathrm{CH}_{2}\right)_{2} \mathrm{OH}\right]^{+}, 282\left[\mathrm{CH}_{3}\left(\mathrm{CH}_{2}\right)_{13}\right.$ $\left.\mathrm{CH}=\mathrm{CHCOH}=\mathrm{CH}-\mathrm{NH}_{2}\right]^{+}, 264,214,157,136 ;{ }^{1} \mathrm{H}$ NMR $(600$ MHz, pyridine- $d_{5}$ ) and ${ }^{13} \mathrm{C}$ NMR (150 MHz, pyridine- $d_{5}$ ): see Table 1.

Methanolysis of 1 . Compound $1(3.0 \mathrm{mg})$ was heated with $1 \mathrm{M} \mathrm{HCL}(5 \mathrm{~mL})$ in $\mathrm{MeOH}(15 \mathrm{~mL})$ for $17 \mathrm{~h}$ at $80^{\circ} \mathrm{C}$. The reaction mixture was extracted three times with $n$-hexane and concentrated under reduced pressure, to give a fatty acid methyl 
ester: ESIMS $m / z 383[\mathrm{M}-\mathrm{H}]^{-}, 365\left[\mathrm{M}-\mathrm{H}-\mathrm{H}_{2} \mathrm{O}\right]^{-}, 351[\mathrm{M}-$ $\left.\mathrm{H}-\mathrm{CH}_{4} \mathrm{O}\right]^{-}, 337\left[\mathrm{M}-\mathrm{H}-\mathrm{C}_{2} \mathrm{H}_{6} \mathrm{O}\right]^{-}, 324\left[\mathrm{M}-\mathrm{H}-\mathrm{COOCH}_{3}\right]^{-}$; ${ }^{1} \mathrm{H}$ NMR $\left(\mathrm{CDCl}_{3}, 250 \mathrm{MHz}\right) \delta 4.18\left(\mathrm{~m}, \mathrm{H}-2^{\prime}\right) 3.77\left(\mathrm{~s}, \mathrm{OCH}_{3}\right)$, 1.23 (br s, H-2' - H-22'), 0.86 (t, $\left.J=6.1 \mathrm{~Hz}, \mathrm{H}-23^{\prime}\right)$.

19-Norergosta-5,7,9,22-tetraene-3 $\beta$-ol (2): White powder; ${ }^{1} \mathrm{H}$ NMR $\left(250 \mathrm{MHz}, \mathrm{CDCl}_{3}\right) \delta_{\mathrm{H}} 0.58(3 \mathrm{H}, \mathrm{s}, \mathrm{H}-18), 0.81(3 \mathrm{H}$, $\mathrm{d}, J=3.7 \mathrm{~Hz}, \mathrm{H}-27), 0.84(3 \mathrm{H}, \mathrm{d}, J=3.7 \mathrm{~Hz}, \mathrm{H}-26), 0.92(3 \mathrm{H}$, d, $J=6.8 \mathrm{~Hz}, \mathrm{H}-28), 1.07$ (3H, d, $J=6.6 \mathrm{~Hz}, \mathrm{H}-21), 2.08(3 \mathrm{H}, \mathrm{s}$, H-19), 3.04 (1H, dd, $J=5.2,16.4 \mathrm{~Hz}, \mathrm{H}-4), 4.15$ (1H, m, H-3), $5.20(1 \mathrm{H}, \mathrm{d}, J=1.7 \mathrm{~Hz}, \mathrm{H}-23), 5.22(1 \mathrm{H}, \mathrm{d}, J=3.8 \mathrm{~Hz}, \mathrm{H}-22)$, $6.64(1 \mathrm{H}, \mathrm{s}, \mathrm{H}-7) ;{ }^{13} \mathrm{C} \mathrm{NMR}\left(63 \mathrm{MHz}, \mathrm{CDCl}_{3}\right) \delta_{\mathrm{C}} 137.8(\mathrm{C}-8)$, 135.5 (C-22), 134.1 (C-6), 132.5 (C-10), 132.0 (C-23), 132.0 (C-9), 129.7 (C-5), 123.8 (C-7), 68.2 (C-3), 55.0 (C-17), 51.8 (C-14), 42.8 (C-24), 41.7 (C-13), 40.5 (C-20), 37.1 (C-12), 36.5 (C-4), 33.0 (C-28), 31.3 (C-2), 29.1 (C-15), 27.5 (C-16), 25.7 (C-11), 24.1 (C-1), 21.0 (C-27), 19.9 (C-21), 19.6 (C-25), 17.6 (C-19), 14.5 (C-26), 11.3 (C-18).

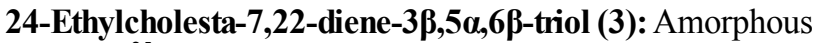
powder; $[\alpha]_{\mathrm{D}}^{25}-4.4^{\mathrm{o}}$ ( $\left.\mathrm{c} 0.1, \mathrm{MeOH}\right)$; HR-EI-MS $m / z 426.3498$ $\left[\mathrm{M}-\mathrm{H}_{2} \mathrm{O}\right]^{+}$, EI-MS $m / z$ (Rel. int., \%): $426\left[\mathrm{M}-\mathrm{H}_{2} \mathrm{O}\right]^{+}$(71), 411 $\left[\mathrm{M}-\mathrm{H}_{2} \mathrm{O}-\mathrm{Me}\right]^{+}(24), 408\left[\mathrm{M}-2 \mathrm{H}_{2} \mathrm{O}\right]^{+}(71), 393\left[\mathrm{M}-2 \mathrm{H}_{2} \mathrm{O}-\right.$ $\mathrm{Me}]^{+}$(90), $287\left[\mathrm{M}-\mathrm{H}_{2} \mathrm{O} \text { - side chain }\right]^{+}(24), 269\left[\mathrm{M}-2 \mathrm{H}_{2} \mathrm{O}-\right.$ side chain $]^{+}$(90), $251\left[\mathrm{M}-3 \mathrm{H}_{2} \mathrm{O}-\text { side chain }\right]^{+}$(100); IR $v_{\max }$ $(\mathrm{KBr}) \mathrm{cm}^{-1}: 3450,1605$ and $1380 ;{ }^{1} \mathrm{H}$ NMR $(250 \mathrm{MHz}$, Pyridine- $\left.d_{5}\right) \delta_{\mathrm{H}} 0.65(3 \mathrm{H}, \mathrm{s}, \mathrm{H}-18), 0.81(3 \mathrm{H}, \mathrm{d}, J=4.6 \mathrm{~Hz}, \mathrm{H}-27)$, $0.85(3 \mathrm{H}, \mathrm{d}, J=1.6 \mathrm{~Hz}, \mathrm{H}-26), 0.94(3 \mathrm{H}, \mathrm{d}, J=6.8 \mathrm{~Hz}, \mathrm{H}-28)$, $1.04(3 \mathrm{H}, \mathrm{d}, J=6.6 \mathrm{~Hz}, \mathrm{H}-21), 1.23(1 \mathrm{H}, \mathrm{m}, \mathrm{H}-14), 1.26,1.71$ (each 1H, m, H-16 $\alpha$, H-16ß), 1.53 (1H, s, H-19), 1.69 (2H, m, H-11), 2.56 (1H, m, H-9), 3.02 (1H, t, $J=12.8 \mathrm{~Hz}, \mathrm{H}-4), 4.32$ $(1 \mathrm{H}, \mathrm{d}, J=4.5 \mathrm{~Hz}, \mathrm{H}-6), 4.83(1 \mathrm{H}, \mathrm{m}, \mathrm{H}-3), 5.17(1 \mathrm{H}, \mathrm{dd}, J=$ $15.0,6.8 \mathrm{~Hz}, \mathrm{H}-22), 5.20$ (1H, dd, $J=15.0,6.8 \mathrm{~Hz}, \mathrm{H}-23)$, $5.80\left(1 \mathrm{H}\right.$, br s, H-7); ${ }^{13} \mathrm{C}$ NMR (63 MHz, Pyridine- $\left.d_{5}\right) \delta_{\mathrm{C}} 140.8$ (C-8), 135.5 (C-22), 131.1 (C-23), 119.8 (C-7), 75.4 (C-5), 73.5 (C-6), 66.9 (C-3), 55.3 (C-17), 54.5 (C-14), 43.0 (C-9, C-13), 42.3 (C-24), 41.3 (C-4), 40.2 (C-20), 39.1 (C-12), 37.3 (C-10), 33.1 (C-25), 32.6 (C-2), 31.9 (C-1), 29.3 (C-28), 27.8 (C-16), 22.7 (C-15), 21.7 (C-11), 20.7 (C-26), 19.4 (C-21), 19.1 (C-27), 18.1 (C-19), 17.1 (C-29), 11.8 (C-18).

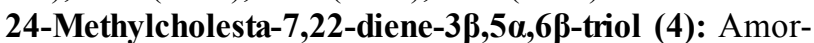
phous powder; $[\alpha]_{\mathrm{D}}^{25}-49.1^{\circ}(\mathrm{c} 0.1, \mathrm{MeOH})$; HR-EI-MS $m / z$ $430.3476\left[\mathrm{M}^{+}\right]$, EI-MS m/z (Rel. int., \%): $430[\mathrm{M}]^{+}$(28), 412 $\left[\mathrm{M}-\mathrm{H}_{2} \mathrm{O}\right]^{+}(37), 394\left[\mathrm{M}-2 \mathrm{H}_{2} \mathrm{O}\right]^{+}$(38), $376\left[\mathrm{M}-3 \mathrm{H}_{2} \mathrm{O}\right]^{+}(45)$, 251 (100), 197 (39); IR $v_{\max }(\mathrm{KBr}) \mathrm{cm}^{-1}: 3500-3100$ (br), 2955, $2871,1726,1658,1458,1384,1371,1254,1162,1032,967$, and $866 ;{ }^{1} \mathrm{H}$ NMR $\left(250 \mathrm{MHz}\right.$, Pyridine- $\left.d_{5}\right) \delta_{\mathrm{H}} 0.65(3 \mathrm{H}, \mathrm{s}, \mathrm{H}-18)$, $0.80(3 \mathrm{H}, \mathrm{d}, J=1.8 \mathrm{~Hz}, \mathrm{H}-27), 0.85(3 \mathrm{H}, \mathrm{d}, J=1.8 \mathrm{~Hz}, \mathrm{H}-26)$, $0.94(3 \mathrm{H}, \mathrm{d}, J=6.8 \mathrm{~Hz}, \mathrm{H}-28), 1.04(3 \mathrm{H}, \mathrm{d}, J=6.6 \mathrm{~Hz}, \mathrm{H}-21)$, 1.24 (1H, m, H-14), 1.27, 1.71 (each 1H, m, H-16 $\alpha, \mathrm{H}-16 \beta), 1.53$ (3H, s, H-19), 1.71 (2H, m, H-11), 2.56 (1H, m, H-9), $3.02(1 \mathrm{H}$,

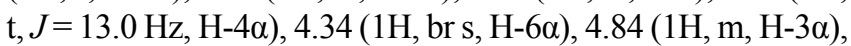
$5.19(1 \mathrm{H}, \mathrm{dd}, J=15.6,6.4 \mathrm{~Hz}, \mathrm{H}-22), 5.28(1 \mathrm{H}$, br d, $J=15.6$ $\mathrm{Hz}, \mathrm{H}-23), 5.74(1 \mathrm{H}, \mathrm{m}, \mathrm{H}-7) ;{ }^{13} \mathrm{C}$ NMR (63 MHz, Pyridine- $\left.d_{5}\right)$ $\delta_{\mathrm{C}} 141.4(\mathrm{C}-8), 136.1$ (C-22), 132.0 (C-23), 120.4 (C-7), 76.0 (C-5), 74.1 (C-6), 67.5 (C-3), 56.0 (C-17), 55.1 (C-14), 43.6 (C-9, C-13), 42.9 (C-24), 41.8 (C-4), 40.8 (C-20), 39.8 (C-12), 37.9 (C-10), 33.7 (C-1), 33.2 (C-25), 32.5 (C-2), 28.4 (C-16), 23.3 (C-15), 22.3 (C-11), 21.3 (C-21), 20.0 (C-27), 19.7 (C-26), 18.7 (C-19), 17.7 (C-28), 12.4 (C-8).
Acknowledgments. This research was supported by the Yeungnam University research grants in 2008.

\section{References}

1. Johnson, T. O.; Ermolieff, J.; Jirousek, M. R. Nat. Rev. Drug Discov. 2002, 1, 696.

2. Pessin, J. E.; Saltiel, A. R. J. Clin. Invest. 2000, 106, 165.

3. Downward, J. Curr. Opin. Cell Biol. 1998, 10, 262.

4. Cheng, A.; Uetani, N.; Simoncic, P. D.; Chaubey, V. P.; Lee-Loy, A.; McGlade, C. J.; Kennedy, B. P.; Tremblay, M. L. Dev. Cell 2002, 2, 497.

5. Kaszubska, W.; Falls, H. D.; Schaefer, V. G.; Haasch, D.; Frost, L.; Hessler, P.; Kroeger, P. E.; White, D. W.; Jirousek, M. R.; Trevillyan, J. M. Mol. Cell Endocrinol. 2002, 195, 109.

6. Zabolotny, J. M.; Haj, F. G.; Kim, Y. B.; Kim, H. J.; Shulman, G. I.; Kim, J. K.; Neel, B. G.; Kahn, B. B. J. Biol. Chem. 2004, 279, 24844.

7. Bialy, L.; Waldmann, H. Angew. Chem. Int. Ed. Engl. 2005, 44, 3814.

8. Ferreira, C. V.; Justo, G. Z.; Souza, A. C. S.; Queiroz, K. C. S.; Zambuzzi, W. F.; Aoyama, H.; Peppelenbosch, M. P. Biochimie 2006, $88,1859$.

9. Na, M. K.; Bae, K. H.; Ahn, J. S.; Min, B. S.; Jin, W. Y. Nat. Prod. Sci. 2008, 14, 143

10. Yuan, D.; Mori, J.; Komatsu, K. I.; Makino, T.; Kano, Y. Biol. Pharm. Bull. 2004, 27, 867.

11. Zhang, J.; Gao, B.; Cun, C.; Lu, X.; Wang, H.; Chen, X.; Tang L. Chin. Med. Sci. J. 1993, 8, 134.

12. Sun, Y.; Yasukawa, K. Bioorg. Med. Chem. Lett. 2008, 18, 3417.

13. Zheng, S. Z.; Yang, H. P.; Ma, X. M.; Shen, X. W. Nat. Prod. Res. 2004, 18, 403.

14. Lu, W.; Adachi, I.; Kano, K.; Yasuta, A.; Toriizuka, K.; Ueno, M.; Horikoshi, I. Chem. Pharm. Bull. 1985, 33, 5083.

15. Ohsawa, T.; Yakawa, M.; Takao, C.; Murayama, M.; Bando, H. Chem. Pharm. Bull. 1992, 40, 143.

16. Miyazaki, T.; Oikawa, N. Chem. Pharm. Bull. 1973, 21, 2545.

17. Bisseret, P.; Adam, H.; Rohmer, M. J. Chem. Soc. Chem. Commun. 1987, 9, 693.

18. Madaio, A.; Piccialli, V.; Sica, D.; Corriero, G. J. Nat. Prod. 1989, 52,952

19. Suri, O. P.; Shah, R.; Satti, N. K.; Suri, K. A. Phytochemistry 1997, 45,1453

20. Hata, K.; Sugawara, F.; Ohisa, N.; Takahashi, S.; Hori, K. Biol. Pharm. Bull. 2002, 25, 1040.

21. Iorizzi, M.; Minale, L.; Riccio, R.; Lee, J. S.; Yasumoto, T. J. Nat. Prod. 1988, 51, 1098

22. Lee, S. H.; Shim, S. H.; Kim, J. S.; Kang, S. S. Arch. Pharm. Res. 2006, 29, 479.

23. Barrero, A. F.; Oltra, J. E.; Poyatos, J. A.; Jimenez, D.; Oliver, E. J. Nat. Prod. 1998, 61, 1491.

24. Singh, P.; Rangaswami, S. Indian J. Chem. 1965, 3, 575.

25. Natori, T.; Morita, M.; Akimoto, K.; Koezuka, Y. Tetrahedron 1994, 50, 2771

26. Lourenco, A.; Lobo, A. M.; Rodriguez, B.; Jimeno, M. L. Phytochemistry 1996, 43, 617.

27. Inagaki, M.; Isobe, R.; Kawano, Y.; Miyamoto, T.; Komori, T.; Higuchi, R. Eur. J. Org. Chem. 1998, 1, 129.

28. Gao, J. M.; Dong, Z. J.; Liu, J. K. Lipids 2001, 36, 175.

29. Meselhy, M. R. Phytochemistry 2003, 62, 213.

30. Udenigwe, C. C.; Ata, A.; Samarasekera, R. Chem. Pharm. Bull. 2007, 55, 442 .

31. Ikeda, I.; Tanaka, K.; Sugano, M.; Vahouny, G. V.; Gallo, L. L. J. Lipid Res. 1988, 29, 1573.

32. Daisy, P.; Jasmine, R.; Ignacimuthu, S.; Murugan, E. Phytomedicine 2009, 16, 252

33. Kizelsztein, P.; Govorko, D.; Komarnytsky, S.; Evans, A.; Wang, Z.; Cefalu, W. T.; Raskin, I. Am. J. Physiol. 2009, 296, E433. 